Gut, 1969, 10, 1015-1019

\title{
Factors which determine the gastric secretory response to 2-deoxy-D-glucose
}

\author{
R. L. HIMSWORTH ${ }^{1}$ AND D. G. COLIN-JONES
}

\begin{abstract}
From the Departments of Medicine and Gastroenterology, University College Hospital Medical School, University Street, London
\end{abstract}

SUMMARY The physiological factors which determine the penetration of the brain by 2-deoxy-Dglucose have been studied and the practical importance of the blood-brain barrier has been demonstrated. The evaluation of the gastric secretory response to 2-deoxy-D-glucose has been shown to depend upon the plasma glucose concentration. It is concluded that because of the hazards and uncertainties in the use of 2-deoxy-D-glucose in man its use is to be deprecated.

The synthetic sugar, 2-deoxy-D-glucose, is an inhibitor of the enzymatic oxidation of glucose. When administered to animals or man 2-deoxy-D-glucose causes activity in the autonomic nervous system similar to that evoked by insulin-induced hypoglycaemia. It has been assumed that both 2-deoxyD-glucose and hypoglycaemia, by acting upon centres within the brain, activate the same nervous mechanisms, the former by inhibiting the utilization of glucose, the latter by diminishing the amount of glucose available for metabolism. The best studied autonomic responses to the administration of 2deoxy-D-glucose are the secretion of adrenaline from the adrenal medulla (Hökfelt and Bydgeman, 1961) and the output of acid from the stomach (Hirschowitz and Sachs, 1965). This paper is concerned with the circumstances which determine the increase in acid secretion from the stomach after the injection of 2-deoxy-D-glucose.

It was found, in the original studies of gastric secretion following 2-deoxy-D-glucose in dogs, that the prior administration of glucose prevented the subsequent increase in gastric acid output whereas glucose given after 2-deoxy-D-glucose did not modify the usual response (Hirschowitz and Sachs, 1965). Likewise it has been shown that dogs made diabetic with alloxan cease to respond to 2-deoxy-D-glucose by secreting acid (Kemp, Herrera, Isaza, and Eisenberg, 1968). Our own studies with another synthetic sugar, 3-O-methylglucose, which may also provoke acid secretion, showed that the onset and rate of gastric secretion could be accounted for by

'Present address: Department of Medicine, College of Physicians and Surgeons of Columbia University, New York, N.Y. 10032. the competition between this sugar and glucose proper for the same transport mechanism at the blood-brain barrier (Colin-Jones and Himsworth, 1969). It therefore seemed likely that the bloodbrain barrier might determine the different responses to 2-deoxy-D-glucose in diabetic or hyperglycaemic animals on the one hand and normal, fasting animals on the other. In the first series of experiments to be described advantage was taken of the previously demonstrated action of the otherwise metabolically inert 3-O-methylglucose to interfere with the transport of other sugars across the blood-brain barrier. In a further series the possibility that the characteristics of the barrier might prevent the easy reversal of the effects of 2-deoxy-D-glucose by the systemic administration of glucose was also investigated.

\section{METHODS}

Gastric acid output was measured in rats by the method which we have described previously and which we have shown to give physiologically consistent results (ColinJones and Himsworth, 1969). Briefly, the hydrogen ions secreted from the stomach of a rat anaesthetized with urethane accumulate in a succinic-propionic buffer which is recirculated through the stomach by way of oesophageal and gastric cannulae. Knowing the volume of buffer in the system, its strength $(M / 200)$ and its characteristics, change in $p \mathrm{H}$, measured by an external electrode, can be recalculated as hydrogen ion excretion. Because urethane anaesthesia activates the sympathetic nervous system and causes the secretion of adrenaline from the adrenal medulla with a consequent rise in plasma glucose concentration, and because a stable plasma glucose concentration was essential in these experiments, the spinal 
cord was cut in all animals at about T2 and the adrenal glands were removed; hydrocortisone, $10 \mathrm{mg}$, was given as replacement therapy. This procedure did not appear to influence the rate of gastric acid secretion in response to appropriate stimuli.

Blood samples were taken from the tip of the tail and the plasma was separated in the manner described previously (Colin-Jones and Himsworth, 1969). Plasma glucose and plasma glucose plus 3-O-methylglucose were measured by an o'toluidine method (Himsworth, 1968a) which is unaffected by the presence of small concentrations of 2-deoxy-D-glucose.

\section{RESULTS}

EFFECTIVE DOSE OF 2-DEOXY-D-GLUCOSE It was found that the minimum amount of 2-deoxy-D-glucose, given as a single dose by rapid intravenous injection, which would invariably cause a sustained secretion of gastric acid in normoglycaemic rats, prepared in the fashion described, was $25 \mathrm{mg}$ (Fig. 1). This dose

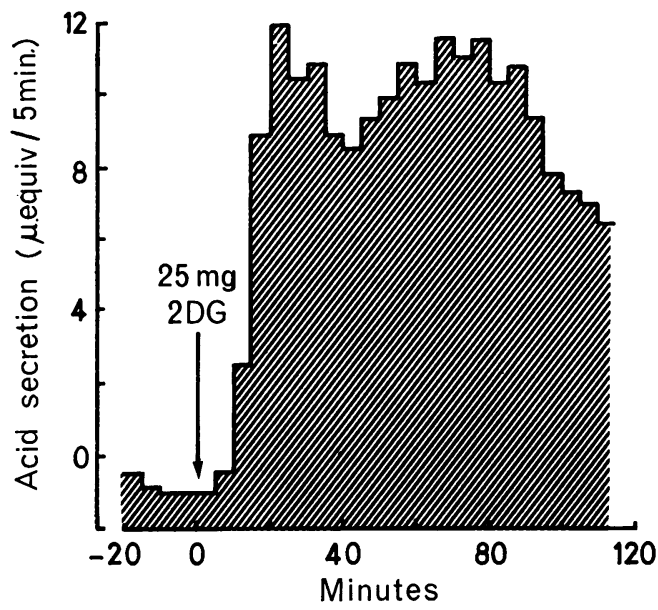

FIG. 1. The effect of a single dose of 2-deoxy-D-glucose (25 mg iv) upon the gastric acid output.

was equivalent to about $80 \mathrm{mg}$ body weight. The latent period between the injection and the onset of acid secretion in these experiments varied from three to 30 minutes, being longest when the plasma glucose concentration was highest (Fig. 2). There is a significant correlation $(P<0.01)$ between the delay in onset and the plasma glucose. Further it was also observed, in confirmation of the observations of previous workers (Hirschowitz and Sachs, 1965; Kemp et al, 1968), that if the initial plasma glucose concentration was greater than $200 \mathrm{mg} / 100$ $\mathrm{ml}$ this dose of 2-deoxy-D-glucose did not cause an increase in gastric acid output.

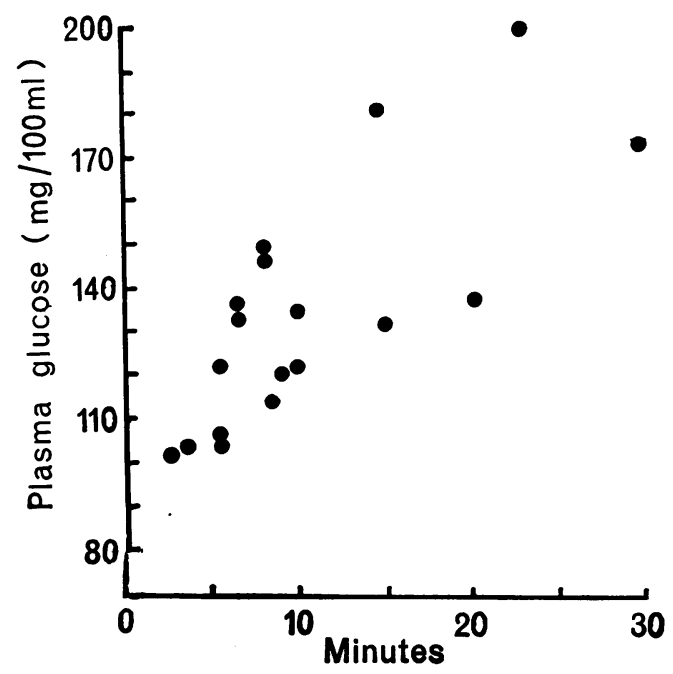

FIG. 2. The relationship between the plasma glucose concentration and the time interval between the administration of 2-deoxy-D-glucose (25 $\mathrm{mg} i v)$ and the onset of gastric acid secretion.

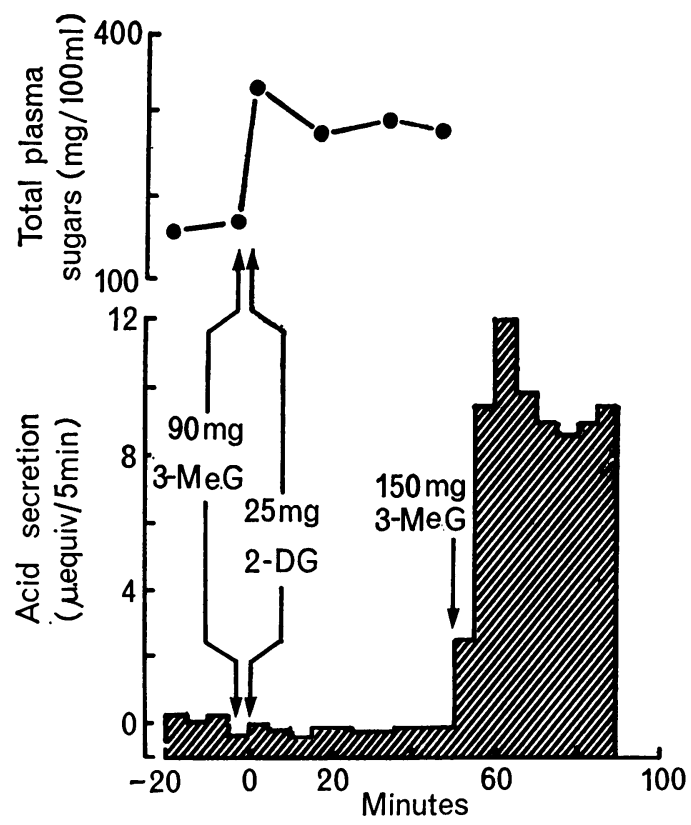

FIG. 3. The effect of 3-O-methylglucose (90 mg iv) upon the plasma total glucose (glucose plus 3-O-methylglucose) and upon the gastric acid secretory response to 2-deoxy$D$-glucose (25 $\mathrm{mg}$ iv). Also shown is the effect of a subsequent large dose of 3-O-methylglucose upon the gastric acid output. 
EFFECT OF 3-O-METHYLGLUCOSE UPON THE ACTION OF 2-DEOXY-D-GLUCOSE It has been shown that large doses of 3-O-methylglucose (sufficient to raise the plasma concentration to over $400 \mathrm{mg} / 100 \mathrm{ml}$ ), by reducing the amount of metabolizable glucose available to the brain, cause an increase in the rate of secretion of gastric acid (Colin-Jones and Himsworth, 1969). Smaller doses, of the order of $90 \mathrm{mg}$ in one dose, are insufficient to produce this effect. It has now been shown in eight experiments, however, that a preliminary dose of $90 \mathrm{mg}$ of 3-O-methylglucose would prevent the gastric secretory response that should follow injection of $25 \mathrm{mg}$ 2-deoxy-Dglucose (Fig. 3). That this prevention of gastric secretion was not due to the inactivation of the responsible mechanism was shown when a further dose of 3-O-methylglucose (enough to bring the plasma concentration above the level that normally elicited a gastric response) was found to be followed by a typical increase in the output of gastric acid.

REVERSAL OF THE EFFECTS OF 2-DEOXY-D-GLUCOSE BY GLUCOSE An established secretion of gastric acid resulting from the administration of 2-deoxy-Dglucose ( $25 \mathrm{mg}$ iv) could always be ended by the intravenous infusion of sufficient glucose (Fig. 4). It was, however, necessary to raise the plasma glucose concentration very considerably to achieve this end. If the infusion of glucose was begun immediately after the onset of acid secretion a mean plasma glucose concentration of $722 \mathrm{mg} / 100 \mathrm{ml}$ (range $489-1,080 / \mathrm{ml}$ in four experiments) was reached before the secretion started to diminish. If, however, the glucose infusion was not begun until one hour after the administration of 2-deoxy-Dglucose the plasma glucose concentration had to be raised to an average of $1,026 \mathrm{mg} / 100 \mathrm{ml}$ (range $764-1,288 \mathrm{mg} / 100 \mathrm{ml}$ in five experiments) before any diminution in acid output became apparent. The difference between these results is significant $(\mathbf{P}<$ 0.01 ). Moreover, acid secretion caused by larger doses of 2-deoxy-D-glucose (200 mg/rat) could also be terminated by the intravenous infusion of glucose but not before a plasma glucose concentration greater than $2,500 \mathrm{mg} / 100 \mathrm{ml}$ was achieved.

\section{DISCUSSION}

It has been shown that 2-deoxy-D-glucose acts upon chemoreceptor cells located in the lateral part of the hypothalamus to bring about the secretion of acid by the stomach (Himsworth and Colin-Jones, 1969). The stimulus to this secretion seems to be a fall in the rate of glucose metabolism below a necessary minimum consequent on the presence within the chemoreceptor cells of sufficient 2-deoxy-

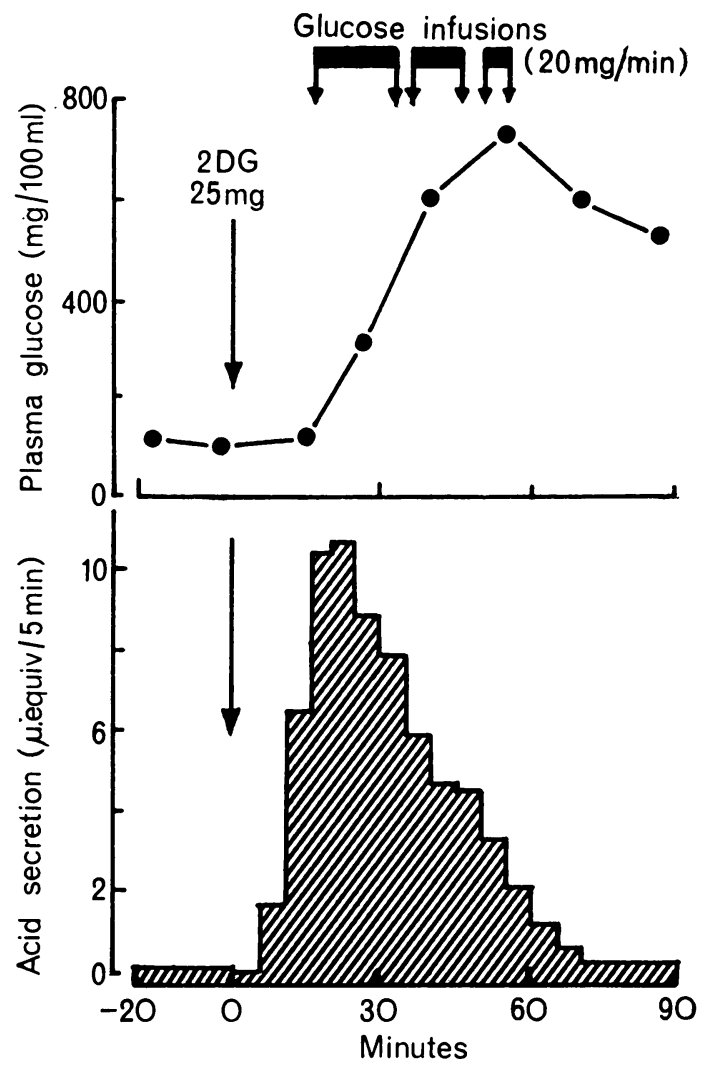

FIG. 4. The effect of an intravenous infusion of glucose (20 $\mathrm{mg} / \mathrm{min}$ ) upon the gastric acid output caused by the administration of 2-deoxy-D-glucose (25 $\mathrm{mg} i \mathrm{v})$. The plasma glucose concentration is also shown.

D-glucose to reduce glycolysis. It would therefore be expected that the factors which influence the access of glucoses to this sensitive region would also influence the response to 2-deoxy-D-glucose.

The brain is enclosed within the physiological blood-brain barrier. This barrier is not freely permeable to glucose; rather this is moved across it by an active transport system of such limited capacity that the rate of transfer of glucose is nearly maximal at fasting plasma glucose concentrations. Passive diffusion of glucose into the brain is insignificant at normal concentrations of plasma glucose although not so if this is grossly elevated artificially (Crone, 1965). Glucose and 3-O-methylglucose use, and have an equal affinity for, the same transport system at the blood-brain barrier (Agnew and Crone, 1967) and, in view of our findings that both glucose and 3-O-methylglucose can prevent 2-deoxyD-glucose exciting the gastric secretory centre in the 
brain, it seems reasonable to conclude that the same transport system is also used by 2-deoxy-D-glucose. The failure of 2-deoxy-D-glucose to affect the gastric acid output in the presence of hyperglycaemia can therefore be readily explained by the competitive exclusion of 2-deoxy-D-glucose from the brain.

If, as the evidence indicates, glucose and 2-deoxyD-glucose are in competition for the same transport system then the higher the plasma glucose concentration the slower will be the accumulation of 2-deoxy-D-glucose in the chemoreceptor cells. One would expect, therefore, what was found, namely, that there is a clear correlation between the height of the plasma glucose concentration, and the length of time between the administration of 2-deoxy-Dglucose and the onset of acid secretion (Fig. 2). This finding further establishes the similarity between the effects of insulin hypoglycaemia and those of 2-deoxy-D-glucose on the intracellular economy. The increased rate of acid secretion after insulin does not occur until the plasma glucose has fallen below a critical level when it is reasonable to suppose that the carbohydrate metabolism within the cell drops to below a threshold value. The correlation between the plasma glucose concentration and the latent period before the onset of gastric secretion after the administration of a standard dose of 2-deoxy-D-glucose is readily explained as being due to the need for the intracellular 2-deoxy-Dglucose to reach a minimum concentration within the chemoreceptor cell before its effects can become manifest. As 2-deoxy-D-glucose causes a competitive block to the enzymatic processes of glycolysis gastric acid secretion depends, not on any direct action of the 2-deoxy-D-glucose as such, but on its indirect effect in reducing intracellular carbohydrate metabolism to below the critical threshold.

In the present experiments acid secretion induced by the minimum dose of 2-deoxy-D-glucose could be stopped only by greatly raising the concentration of glucose in the plasma and thus increasing the passive component of glucose transfer into the cells of the brain. The longer the interval between the administration of 2-deoxy-D-glucose and the start of glucose infusion, which will inhibit the entry of further 2-deoxy-D-glucose by increasing the competition at the blood-brain barrier, the more 2-deoxyD-glucose will have entered the brain and therefore, as was found, a higher plasma glucose concentration will be needed if the intracellular metabolic block is to be overcome. Likewise, the larger the dose of 2-deoxy-D-glucose the higher the plasma glucose concentration necessary to overcome both the barrier to glucose penetration of the brain and the intracellular inhibition of glucose utilization.

The effect of 2-deoxy-D-glucose upon the gastric acid output therefore depends neither upon the dose of 2-deoxy-D-glucose alone nor the plasma concentration of 2-deoxy-D-glucose, nor the concentration of plasma glucose but upon the relationship between these factors.

The administration of 2-deoxy-D-glucose has been advocated in testing for the completeness of operative vagotomy. It has been suggested that its use may have some advantages over the more conventional assessment of acid output during insulininduced hypoglycaemia (Thomas and Duthie, 1968). We believe that the use of 2-deoxy-D-glucose for this purpose has some disadvantages and, in addition, is not without hazard.

In practice 2-deoxy-D-glucose will often be given in the expectation that acid will not be secreted. The interpretation of a negative response in a person who has undergone vagotomy depends upon the assumption that the dose given would in a normal person have provoked a secretion of acid. But, as we have seen, a positive result depends not only on the dose given but also on the particular concentration of glucose in the plasma. A standard dose of 2-deoxy-D-glucose that could be counted on to cause gastric secietion in all patients is thus not feasible. At best a ratio of dosage to the current plasma glucose concentration would have to be worked out and applied individually to each case.

The hazard in the use of 2-deoxy-D-glucose for the purposes of investigation in man is twofold. First, because of the characteristics of the bloodbrain barrier the cells of the brain become progressively more accessible to 2-deoxy-D-glucose as the plasma glucose concentration falls below normal. Therefore in any subject with hypoglycaemia a greater proportion of an administered dose of 2deoxy-D-glucose would be expected to enter the brain than in normoglycaemic subjects. As 2-deoxy-Dglucose inhibits glucose metabolism throughout the central nervous system such individuals might develop a state of depressed cerebral function equivalent to profound hypoglycaemia. Secondly, the effects of 2-deoxy-D-glucose can be reversed only slowly by raising and maintaining the plasma concentration of glucose at heroic levels. The high glucose concentrations required will cause marked hyperosmolarity which in itself may be deleterious.

2-Deoxy-D-glucose should clearly never be given to any patient unless the whole blood or plasma glucose concentration is known to be normal at the time of administration, nor should the dose of 2deoxy-D-glucose be increased if no effect is obtained. In our opinion, its use to evoke gastric acid secretion is better abandoned in favour of the safer and better understood method of provocation by insulin hypoglycaemia. 
We are grateful to Professor Sir Max Rosenheim, Professor C. G. Clark, and Dr J. E. Lennard-Jones for their encouragement. Miss M. Cornelis gave skilled technical assistance. One of us (D.G.C-J.) is in receipt of a grant from the Medical Research Council. the other has financial help from the Wellcome Trust.

\section{REFFRENCES}

Agnew, W. F., and Crone, C. (1967). Permeability of brain capillaries to hexoses and pentoses in rabbit. Acta physiol. scand., 70, 168-175.

Colin-Jones, D. G., and Himsworth, R. L. (1969). The secretion of gastric acid in response to a lack of metabolizable glucose. J. Physiol. (Lond.), 202, 97-109.

Crone, C. (1965). Facdilitated transfer of glucose from blood into brain tissue. Ibid, 181, 103-113.
Himsworth, R. L., ( 2968 a). Compensatory reactions to lack of metabolizable glucose. Ibid, 198, 451-465.

-, (1968b). Interference with the metabolism of glucose by a non-metabolizable hexose (3-methylglucose). Ibid, 198, 467-477.

- and Colin-Jones, D. G. (1969). The location of the chemoreceptor controlling gastric acid secretion during hypoglycaemia. Clin. Sc., 37, 568

Hirschowitz, B. I., and Sachs, G. (1965). Vagal gastric secretory stimulation by 2-deoxy-D-glucose. Amer. J. Physiol., 209, 452-460.

Hökfelt, B., and Bydgeman, S. (1961). Increased adrenaline production following administration of 2-deoxy-D-glucose in the rat. Proc. Soc. exp. biol. (N.Y), 106, 537-539.

Kemp, D. R., Herrera, F., Isaza, J., and Eisenberg, M. M. (1968). On the critical nature of blood sugar levels in the vagal stimulation of gastric acid secretion in normal and diabetic dogs. Surgery, 64, 958-966.

Thomas, D. G., and Duthie, H. L. (1968). Use of 2-deoxy-D-glucose to test for the completeness of surgical vagotomy. Gut, 9, 125-128. 